\title{
Application of Resolvability Technique to Investigate the Different Polyphenyl Structures for Polymer Industry
}

\author{
Muhammad Faisal Nadeem (D), ${ }^{1}$ Mohsan Hassan, ${ }^{1}$ Muhammad Azeem (D), \\ Salah Ud-Din Khan, ${ }^{3}$ Mohammed Rafi Shaik ${ }^{1},{ }^{4}$ Mohammed A. F. Sharaf, ${ }^{5}$ \\ Abdelatty Abdelgawad, ${ }^{5}$ and Emad Mahrous Awwad ${ }^{6}$ \\ ${ }^{1}$ Department of Mathematics, COMSATS University Islamabad, Lahore Campus, Lahore, Pakistan \\ ${ }^{2}$ Department of Aerospace Engineering, Faculty of Engineering, University Putra Malaysia, Seri Kembangan, Malaysia \\ ${ }^{3}$ Sustainable Energy Technologies (SET) Center, College of Engineering, King Saud University, P O. Box 800, \\ Riyadh 11421, Saudi Arabia \\ ${ }^{4}$ Department of Chemistry, College of Science, King Saud University, P.O. Box 2455, Riyadh 11451, Saudi Arabia \\ ${ }^{5}$ Department of Industrial Engineering, College of Engineering, King Saud University, P.O. Box 800, Riyadh-11421, Saudi Arabia \\ ${ }^{6}$ Department of Electrical Engineering, College of Engineering, King Saud University, P.O. Box 800, Riyadh, Saudi Arabia
}

Correspondence should be addressed to Mohammed Rafi Shaik; mrshaik@ksu.edu.sa

Received 29 November 2020; Revised 13 December 2020; Accepted 31 March 2021; Published 20 April 2021

Academic Editor: Leena Gupta

Copyright (c) 2021 Muhammad Faisal Nadeem et al. This is an open access article distributed under the Creative Commons Attribution License, which permits unrestricted use, distribution, and reproduction in any medium, provided the original work is properly cited.

Polyphenyl is used in a variety of applications including high-vacuum devices, optics, and electronics, and in high-temperature and radiation-resistant fluids and greases, it has low volatility, ionizing radiation stability, and high thermal-oxidative properties. The structure of polyphenyls can be represented using a molecular graph, where atoms represent vertices and bonds between atom edges. In a chemical structure, an item/vertex $v$ resolves two items $v_{1}$ and $v_{2}$ if $\mathrm{d}\left(v_{1}, v\right) \neq \mathrm{d}\left(v_{2}, v\right)$; similarly, the ordered subset $\phi$ of vertices resolves each pair of distinct vertices named as the resolving set, and its minimum cardinality is described as metric dimension. In the pharmaceutical industry, the competition to find new chemical entities for treating a disease dictates larger project teams that encompass more extensive and diverse synthetic efforts directed at increasingly complicated activity spectra. In this paper, we determine the metric dimension of para-, meta-, and ortho-polyphenyl structures, which are used for structureactivity analysis of these polyphenyl structures.

\section{Introduction}

Chemists require the mathematical representation of a chemical compound to work with the chemical structure. In a chemical structure, a set of selected atoms gave mathematical representations so that it gave distinct representations to distinct atoms of the structure. The chemical structure can be defined in the form of vertices, which mentions the atom and edges indicate the bonds types, respectively. Thus, a graph-theoretic analysis of this idea yields the representations of all vertices in a structure in such a way that different vertices have distinct representations with respect to some specific atoms of that structure. The following are some mathematical definitions to indicate these concepts.

In 1975, the concept of locating set was proposed by Slater [1] and called the minimum cardinality of a locating set of a graph locating number. On the same pattern, in 1976 , the idea of metric dimension of a graph was individually introduced by Harary and Melter in [2], and these time metric generators were named as resolving sets. Members of metric basis set were assigned as a sonar or loran station [1].

A connected, simple graph $G(V, E)$ with $V$ is the set of vertices (also can say atoms), and $E$ is the set of edges (bond types); the distance between two vertices/bonds $a_{1}, a_{2} \in V$ is 
the length of geodesic between them and denoted by $\mathrm{d}\left(a_{1}, a_{2}\right)$. Let $\phi=\left\{\phi_{1}, \phi_{2}, \ldots, \phi_{l}\right\}$ be an order subset of vertices belonging to a graph $G$ and $a$ be a vertex. The representation $r(a \mid \phi)$ of $a$ corresponding to $\phi$ is the $l$-tuple $\left(\mathrm{d}\left(a, \phi_{1}\right), \mathrm{d}\left(a, \phi_{2}\right), \mathrm{d}\left(a, \phi_{3}\right), \ldots, \mathrm{d}\left(a, \phi_{l}\right)\right)$, where $\phi$ is called a resolving set [2] or locating set [1], if every vertex of $G$ is uniquely determined by its distances from the vertices of $\phi$ or, on the contrary, if different vertices of $G$ have unique representations with respect to $\phi$. The minimum cardinality of the resolving set $\phi$ is called the metric dimension of $G$, and it is denoted by $\operatorname{dim}(G)$ [1]. For a given ordered set of vertices $\phi=\left\{\phi_{1}, \phi_{2}, \ldots, \phi_{l}\right\} \subset V$, the $c$ th location of $r(a \mid \phi)=$ 0 if and only if $a=\phi_{c}$. Thus, to verify that $\phi$ is a resolving set, it is enough to show that $r\left(a_{1} \mid \phi\right) \neq r\left(a_{2} \mid \phi\right)$ for every possible distinct pair of vertices $a_{1}, a_{2} \in V(G) \backslash \phi$.

Metric dimension of a graph or a structure is a resolvability parameter that has been applied in numerous applications of graph theory, for the drug discovery in pharmaceutical chemistry [3, 4], robot navigation [5], combinatorial optimization concept studied in [6], various coin weighing problems $[7,8]$, and utilization of the idea in pattern recognition and processing of images, few of which also associate with the use in hierarchical data structures [1].

Due to numerous uses of resolvability parameters in the chemical field, many works have been done with graph perspectives, and metric dimension is also considered important to study different structures with it, such as the structure of $\mathrm{H}$-naphtalenic and $V C_{5} C_{7}$ nanotubes discussed with metric concept [9], some upper bounds of cellulose network considering metric dimension as a point of discussion [10], resolving sets of silicate star determined in [11], metric basis of $2 \mathrm{D}$ lattice of alpha-boron nanotubes discussed with specific applications [12], and sharps bound on the metric dimension of honeycomb and its related network [13]; for more interesting literature work on metric dimension, metric basis, resolving set, and other resolvability parameters, refer to [13-28].

\section{Results of Polyphenyl Chemical Networks}

In the results of this article, we discuss the metric dimension of para-, meta-, and ortho-polyphenyl chemical networks constructed by different polygons. Usually, the networks are made up with the chain of hexagons using chemical operations ortho, para, and meta; in this work, we extend this to any order of polygons. Moreover, using $\eta=6$ with arbitrary $h$ in Theorems 1-5, we can produce the para-, meta-, and ortho-polyphenyl chain of hexagons and retrieve its corresponding metric dimension as well.

2.1. Metric Dimension of $\mathbf{O}(\eta, \mathbf{h})$. Let $O(\eta, h)$ be a connected graph of ortho-polyphenyl network of cycle graph $C_{\eta}$, and $h$ are the copies of cycle graph with order $\lambda=\eta h$ and size $h(\eta+1)-1$. For the following theorems, Figure 1 shows the resolving set in dark black vertices.

Theorem 1. If $\eta \geq 3$ and $h=2$, then $\operatorname{dim}(O(\eta, 2))$ is 2 .

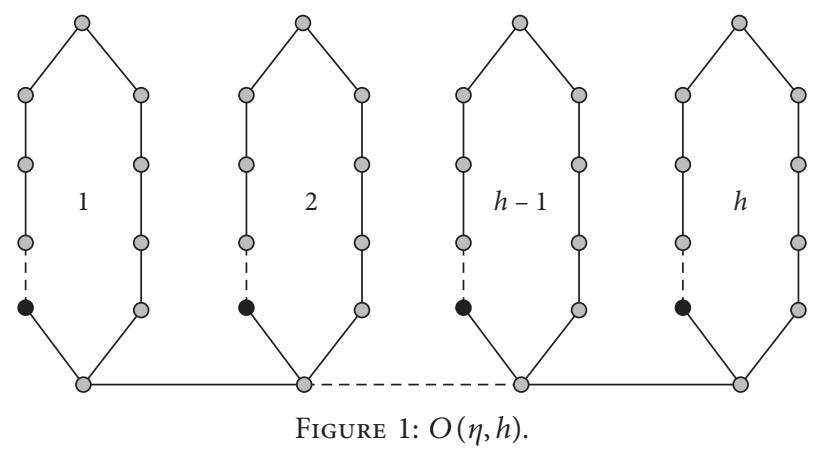

Proof. To prove that $\operatorname{dim}(O(\eta, 2)) \leq 2$, for this assume, a resolving set $\phi=\left\{v_{1}, v_{\eta+1}\right\}$. We construct the following cases on vertex set of $O(\eta, 2)$ :

$$
r_{1}\left(v_{\zeta} \mid \phi\right)=\left(\begin{array}{ll}
\zeta-1 ; & \text { if } \zeta=1,2,3, \ldots, \frac{\eta}{2}+1 ; \\
\eta-\zeta+1 ; & \text { if } \zeta=\frac{\eta}{2}+2, \ldots, \eta \\
\zeta-\eta+2 ; & \text { if } \zeta=\eta+1, \eta+2, \ldots, \eta+\frac{\eta}{2} ; \\
2 \eta-\zeta+2 ; & \text { if } \zeta=\eta+\frac{\eta}{2}+1, \ldots, 2 \eta .
\end{array}\right.
$$

Second vector representations are as follows:

$$
r_{2}\left(v_{\zeta} \mid \phi\right)= \begin{cases}\zeta+2 ; & \text { if } \zeta=1,2,3, \ldots, \frac{\eta-1}{2}, \\ \eta-\zeta+2 ; & \text { if } \zeta=\frac{\eta-1}{2}+1, \ldots, \eta, \\ \zeta-\eta ; & \text { if } \zeta=\eta+1, \eta+2, \ldots, \eta+\frac{\eta+2}{2}, \\ 2 \eta-\zeta+1 ; & \text { if } \zeta=\eta+1+\frac{\eta+2}{2}, \eta+2+\frac{\eta+2}{2}, \ldots, 2 \eta .\end{cases}
$$

Hence, it follows from the above arguments in the form of representation that $\operatorname{dim}(O(\eta, 2)) \leq 2$ because all the vertices of $O(\eta, 2)$ have unique representations with respect to resolving set $\phi$.

For reverse inequality that $\operatorname{dim}(O(\eta, 2)) \geq 2$, by contradiction, our assertion becomes $\operatorname{dim}(O(\eta, 2))<2$, implying that $\operatorname{dim}(O(\eta, 2))=1$, and it is not possible because only the path graph exists having the metric dimension 1. All discussion concludes that when $\eta \geq 3$ and $h=2$,

$$
\operatorname{dim}(O(\eta, 2))=2 \text {. }
$$

Theorem 2. If $\eta, h \geq 3$, then $\operatorname{dim}(O(\eta, h))$ is $h$.

Proof. To show that $\operatorname{dim}(O(\eta, h))=h$, we will apply the induction method on $h$ the number of copies of base graph. 
The base case for $h=2$ is proved in Theorem 1; now, assume that the assertion is true for $h=m$ :

$$
\operatorname{dim}(O(\eta, m))=m \text {. }
$$

We will show that it is true for $h=m+1$. Suppose

$$
\operatorname{dim}(O(\eta, m+1))=\operatorname{dim}(O(\eta, m))+\operatorname{dim}(O(\eta, 2))-1 .
$$

Using equations (3) and (4) in equation (5), we will get

$$
\operatorname{dim}(O(\eta, m+1))=m+2-1=m+1 .
$$

Hence, the result is true for all positive integers $h \geq 3$.

2.2. Metric Dimension of $M(\eta, h)$. Let $M(\eta, h)$ be a connected graph of meta-polyphenyl network of cycle graph $C_{\eta}$, and $h$ are the copies of cycle graph with order $\lambda=\eta h$ and size $h(\eta+1)-1$. For the following theorems, Figure 2 shows the resolving set in dark black vertices.

Theorem 3. If $\eta \geq 4$ (even) and $h=2$, then $\operatorname{dim}(M(\eta, 2))$ is 2 .

Proof. To prove that $\operatorname{dim}(M(\eta, 2)) \leq 2$, we construct a resolving set $\phi=\left\{v_{1}, v_{\eta+2}\right\}$ from the vertex set of $M(\eta, 2)$. We assume the following cases on vertex set of $M(\eta, 2)$ :

$$
\begin{aligned}
& r\left(v_{\zeta} \mid \phi\right)=\left(\frac{\zeta+1}{2}, \frac{\eta+4}{2}-\frac{\zeta}{2}\right), \quad \zeta=1,3,5, \ldots, \eta-1, \\
& r\left(v_{\zeta} \mid \phi\right)=\left(\frac{\zeta-1}{2}, \frac{\eta+2}{2}-\frac{\zeta-1}{2}\right), \quad \zeta=2,4,6, \ldots, \eta, \\
& r\left(v_{\zeta} \mid \phi\right)=\left(\frac{\zeta-1}{2}, \frac{\zeta-\eta+1}{2}\right), \quad \zeta=\eta+1, \eta+3, \eta+5, \ldots, 2 \eta-1, \\
& r\left(v_{\zeta} \mid \phi\right)=\left(\frac{\zeta}{2}, \frac{\zeta-\eta-2}{2}\right), \quad \zeta=\eta+2, \eta+4, \eta+6, \ldots, 2 \eta .
\end{aligned}
$$

Hence, it follows from the above discussion that $\operatorname{dim}(M(\eta, 2)) \leq 2$ because all the vertices of $M(\eta, 2)$ have unique representations with respect to resolving set $\phi$.

For converse $\operatorname{dim}(M(\eta, 2)) \geq 2$, we use contradiction, and $\operatorname{dim}(M(\eta, 2))=1$ is not possible because only the path

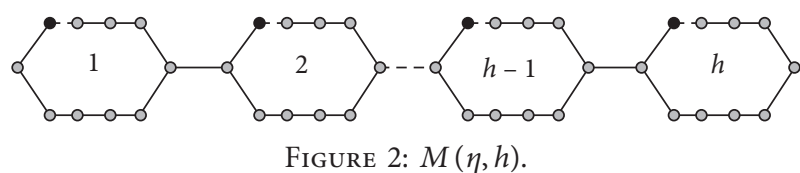

graph exists having the metric dimension 1. All discussion concludes that when $\eta \geq 4$ (even) and $h=2$,

$$
\operatorname{dim}(M(\eta, 2))=2 \text {. }
$$

Theorem 4. If $\eta \geq 4$ (even) and $h \geq 3$, then $\operatorname{dim}(M(\eta, h))$ is $h$.

Proof. To show that $\operatorname{dim}(M(\eta, h))=h$, we will apply the induction method on $h$ showing the copies of base graph. The base case for $h=2$ is proved in Theorem 3; now, assume that the assertion is true for $h=m$ :

$$
\operatorname{dim}(M(\eta, m))=m \text {. }
$$

We will show that it is true for $h=m+1$. Suppose

$$
\operatorname{dim}(M(\eta, m+1))=\operatorname{dim}(M(\eta, m))+\operatorname{dim}(M(\eta, 2))-1 .
$$

Using equations (8) and (9) in equation (10), we have

$$
\operatorname{dim}(M(\eta, m+1))=m+2-1=m+1 .
$$
$h \geq 3$.

Hence, the result is true for all positive integers

2.3. Metric Dimension of $L(\eta, h)$. Let $L(\eta, h)$ be a connected graph of para-polyphenyl network of cycle graph $C_{\eta}$, and $h$ are the copies of cycle graph with order $\lambda=\eta h$ and size $h(\eta+1)-1$. For the following theorems, vertices are labeled, as shown in Figure 3; moreover, it also shows the resolving set in dark black vertices.

Theorem 5. If $\eta \geq 5$ and $h \geq 2$, then $\operatorname{dim}(L(\eta, h))$ is 2 .

Proof. Firstly, we prove that $\operatorname{dim}(L(\eta, h)) \leq 2$; for this construction, a resolving set $\phi=\left\{v_{1}, v_{\lambda-\eta+1}\right\}$ from the vertex set of $L(\eta, h)$. We assume the following cases on vertex set of $G$ and on the copies of cycle graph, i.e., $h$ :

$$
r_{1}\left(v_{\zeta} \mid \phi\right)= \begin{cases}\zeta-1, & \text { if } \zeta=1,2,3, \ldots, \frac{\eta+1}{2}, \\ \eta-\zeta+1, & \text { if } \zeta=\frac{\eta+1}{2}+1, \frac{\eta+1}{2}+2, \ldots, \eta \\ 3 \frac{\zeta}{\eta}+\zeta-1-\eta \frac{\zeta}{\eta}, & \text { if } \zeta \equiv 1,2,3, \ldots, \frac{\eta-1}{2}(\bmod \eta), \\ \eta \frac{\zeta}{\eta}+2-\zeta, & \text { if } \zeta \equiv 0, \frac{\eta-1}{2}+1, \frac{\eta-1}{2}+2, \ldots, \eta-1(\bmod \eta) .\end{cases}
$$




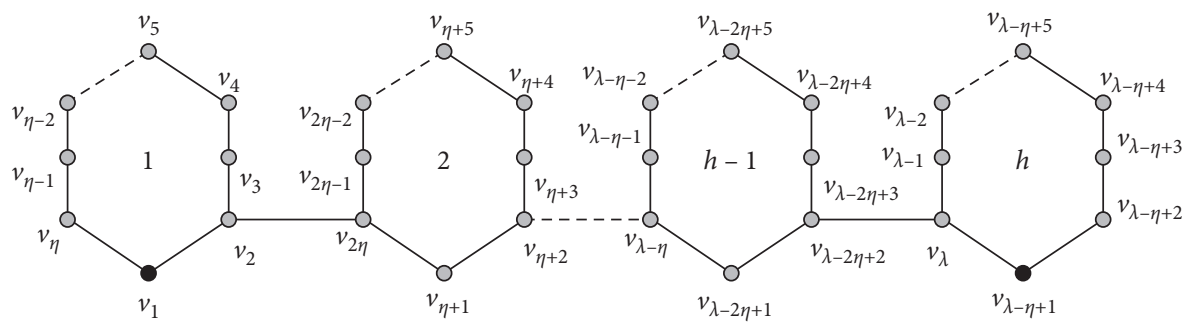

Figure 3: $L(\eta, h)$.

If $\zeta \equiv 0,((\eta-1) / 2)+1,((\eta-1) / 2)+2, \ldots, \eta-1(\bmod$ $\eta)$ and $\zeta>2 \eta$,

$$
\begin{aligned}
r_{1}\left(v_{\zeta} \mid \phi\right)= & \eta \frac{\zeta+((\eta-1) / 2)+1}{\eta} \\
& -\zeta+2+3\left(\frac{\zeta+((\eta-1) / 2)+1}{\eta}-2\right)
\end{aligned}
$$

Second vector representations are as follows:

Case $1 . h=2$ :

$$
r_{2}\left(v_{\zeta} \mid \phi\right)=\left(\begin{array}{ll}
3, & \text { if } \zeta=1 ; \\
\zeta, & \text { if } \zeta=2,3, \ldots, \frac{\eta+2}{2}, \\
\eta-\zeta+4, & \text { if } \zeta=\frac{\eta+2}{2}+1, \ldots, \eta .
\end{array}\right.
$$

Case 2. $h \geq 3$ :

$$
r_{2}\left(v_{\zeta} \mid \phi\right)=\left(\begin{array}{ll}
3\left(\frac{\eta(h-1)+1}{\eta}-1\right)+\zeta, & \text { if } \zeta=1, \\
3\left(\frac{\zeta}{\eta}-1\right)+\zeta, & \text { if } \zeta=2,3, \ldots, \frac{\eta+2}{2}, \\
\eta-\zeta+4+3\left(\frac{\eta(h-1)+1}{\eta}-1\right), & \text { if } \zeta=\frac{\eta+2}{2}+1, \ldots, \eta
\end{array}\right.
$$

Subcase 2.1. If $\zeta=\eta(h-1)+1, \eta(h-1)+2, \ldots$, $\eta(h-1)+(\eta / 2)$,

$$
r_{2}\left(v_{\zeta} \mid \phi\right)=\zeta-\eta(h-1)-1 .
$$

Subcase 2.2. If $\zeta=\eta(h-1)+(\eta / 2)+1, \eta(h-1)+$ $(\eta / 2)+2, \ldots, \eta h$

$$
r_{2}\left(v_{\zeta} \mid \phi\right)=\eta h-\zeta+1
$$

Subcase 2.3. If $\zeta \equiv 2,3, \ldots,((\eta+2) / 2)(\bmod \eta)$, and $\zeta \geq \eta+1$,

$$
r_{2}\left(v_{\zeta} \mid \phi\right)=3\left(h-\frac{\zeta-1}{\eta}-2\right)+2+\zeta-\left(2+\eta \frac{\zeta}{\eta}\right) .
$$

Subcase 2.4. If $\zeta \equiv 0,((\eta+2) / 2)+1, \ldots, \eta-1$ $(\bmod \eta)$, and $\zeta \geq \eta+1$,

$$
r_{2}\left(v_{\zeta} \mid \phi\right)=3\left(h-\frac{\zeta-1}{\eta}-2\right)+4-\zeta+\eta \frac{\zeta+((\eta+2) / 2)}{\eta} .
$$

Subcase 2.5. If $\zeta \equiv 1(\bmod \eta)$, and $\zeta \geq \eta+1$,

$$
r_{2}\left(v_{\zeta} \mid \phi\right)=3\left(h-\frac{\zeta}{\eta}-1\right) .
$$

Hence, it follows from the above discussion that $\operatorname{dim}(L(\eta, h)) \leq 2$ because all the vertices of $L(\eta, h)$ have unique representations with respect to resolving set $\phi$.

For reverse inequality that $\operatorname{dim}(L(\eta, h)) \geq 2$, by contradiction, our assertion becomes $\operatorname{dim}(L(\eta, h))<2$, implying that $\operatorname{dim}(L(\eta, h))=1$, and it is not possible because only the path graph exists having the metric dimension 1. All discussion concluding that when $\eta \geq 5$ and $h \geq 2$,

$$
\operatorname{dim}(L(\eta, h))=2 \text {. }
$$

2.4. Metric Dimension of $L_{\mathbf{S}}(\eta, h)$. Let $L_{S}(\eta, h)$ be a connected graph of para-polyphenyl network of sun graph $S_{\eta}$, where $h$ are the copies of sun graph and $\eta$ is the order of interior cycle of sun graph. The order and size of network are 
$\left|V\left(L_{S}(\eta, h)\right)\right|=\lambda=2 \eta h$ and $\left|E\left(L_{S}(\eta, h)\right)\right|=h(2 \eta+1)-1$, respectively. The vertices are labeled, as shown in Figure 4.

Theorem 6. If $\eta \geq 5$ (odd) and $h \geq 2$, then $\operatorname{dim}\left(L_{S}(\eta, h)\right)$ is 2 .

Proof. To prove that $\operatorname{dim}\left(L_{S}(\eta, h)\right) \leq 2$, for this construct, a resolving set $\phi=\left\{v_{((\eta+3) / 2)}, v_{\xi}\right\}$, where $\xi=((\eta(4 h-3)-1) /$ 2 ) from the vertex set of $L_{S}(\eta, h)$. We assume the following cases on vertex set of $L_{S}(\eta, h)$ :

Case $1.1 \leq \zeta \leq 2 \eta$ :

$$
r_{1}\left(v_{\zeta} \mid \phi\right)= \begin{cases}\frac{\eta+3}{2}-\zeta, & \text { if } \zeta=1,2, \ldots, \frac{\eta+3}{2}, \\ \zeta-\frac{\eta+3}{2}, & \text { if } \zeta=\frac{\eta+5}{2} \frac{\eta+5}{2}, \ldots, \eta, \\ \frac{\eta+1}{2}, & \text { if } \zeta=\eta+1, \\ \frac{3 \eta+5}{2}-\zeta, & \text { if } \zeta=\eta+2, \eta+3, \ldots, \frac{3 \eta+3}{2}, \\ \zeta-\frac{3 \eta+1}{2}, & \text { if } \zeta=\frac{3 \eta+5}{2}, \frac{3 \eta+7}{2}, \ldots, 2 \eta .\end{cases}
$$

Case 2. If $\zeta \equiv((\eta+1) / 2),((\eta+3) / 2), \ldots, \eta(\bmod 2 \eta)$, $\zeta \geq((5 \eta+1) / 2)$

$r_{1}\left(v_{\zeta} \mid \phi\right)=\left(\frac{\eta-1}{2}+3 \eta+2\left(\frac{\zeta-1}{3 \eta}\right) \eta-\zeta+3 \frac{\zeta}{2 \eta}+2\left(\frac{\zeta}{2 \eta}\right)-1\right)$.

Case 3. If $\zeta \equiv 1,2, \ldots,((\eta-1) / 2)(\bmod 2 \eta), \zeta \geq 2 \eta+1$,

$r_{1}\left(v_{\zeta} \mid \phi\right)=\left(\frac{\eta-1}{2}+\zeta+5 \frac{\zeta}{2 \eta}-\frac{\eta(4 h-3)-1}{2}+\frac{\eta-5}{2}\right)$.

Case 4. If $\zeta \equiv \eta+1, \eta+2, \ldots,(3 \eta-1 / 2)(\bmod 2 \eta)$, $\zeta \geq 3 \eta+1$,

$r_{1}\left(v_{\zeta} \mid \phi\right)=\left(\zeta-\frac{\eta(4((\zeta / 2 \eta)+1)-3)-1}{2}+3 \frac{\zeta}{2 \eta}+2\left(\frac{\zeta}{2 \eta}-1\right)\right)$.

Case 5. If $\zeta \equiv 0,((3 \eta+1) / 2),((3 \eta+3) / 2), \ldots, 2 \eta-1$ $(\bmod 2 \eta), \zeta \geq((7 \eta+1) / 2)$,

$$
r_{1}\left(v_{\zeta} \mid \phi\right)=\left(\frac{\eta-1}{2}+4 \frac{\zeta-1}{2 \eta}+2\left(\frac{\zeta}{2 \eta}-1\right)-\zeta+\eta\left(2 \frac{\zeta-1}{2 \eta} 2\right)-\frac{\zeta-\eta-1}{3 \eta}+z\right)
$$

where $z=-2$ when $\zeta \equiv 0(\bmod 2 \eta)$ and $\zeta \geq 4 \eta$; otherwise, $z=0$.

Case $1.1 \leq \zeta \leq 3 \eta+1$ :

The representations of all vertices with respect to the second vertex of resolving set are as follows:

$$
r_{2}\left(v_{\zeta} \mid \phi\right)= \begin{cases}\zeta-\xi, & \text { if } \zeta=\xi+1, \xi+2, \ldots, \frac{2 \xi+\eta-1}{2}, \\ \frac{\eta+1}{2}, & \text { if } \zeta=\frac{2 \xi+\eta+1}{2}, \\ \xi-\zeta, & \text { if } \zeta=\frac{2 \xi+\eta+1}{2}, \frac{2 \xi+\eta+1}{2}+1, \ldots, \xi, \\ \zeta-\xi-\eta+1, & \text { if } \zeta=\xi+\eta, \xi+\eta+1, \xi+\eta+2, \ldots, \frac{2 \xi+3 \eta-1}{2}, \\ \frac{\eta+3}{2}, & \text { if } \zeta=\frac{2 \xi+3 \eta+1}{2}, \\ \frac{\eta-1}{2}, & \text { if } \zeta=3 \eta+1 .\end{cases}
$$




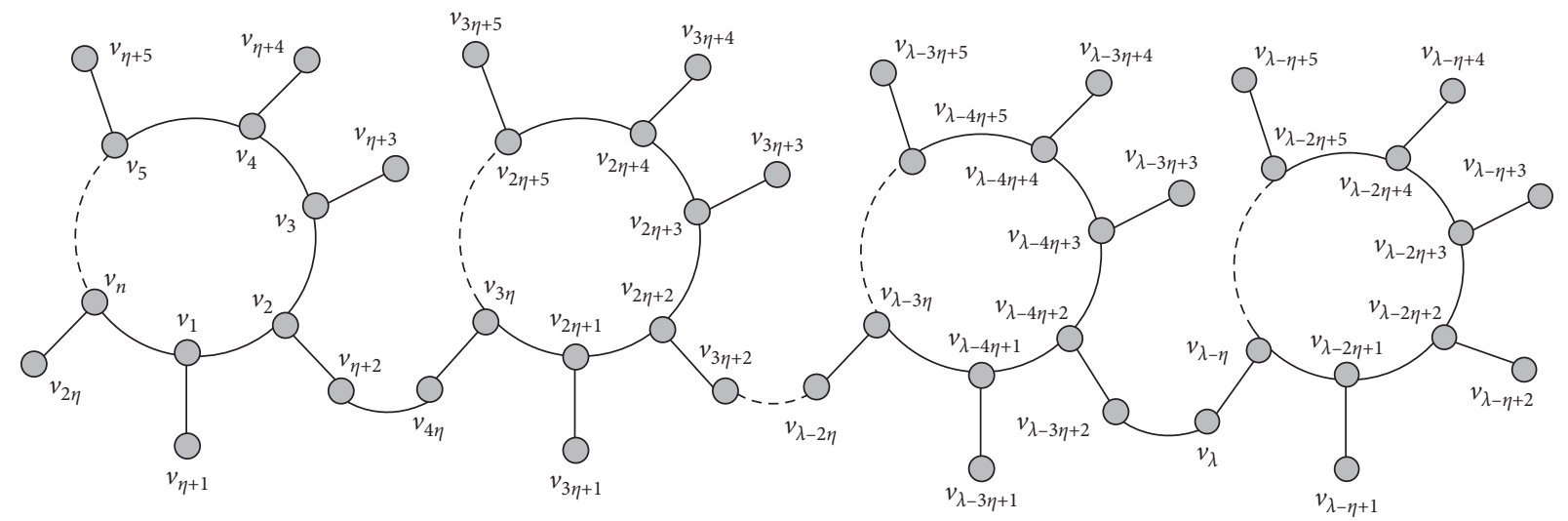

Figure 4: $L_{S}(\eta, h)$.

Case 2 . If $\zeta \equiv 2,3,4, \ldots,((\eta+3) / 2)(\bmod 2 \eta)$,

$$
r_{2}\left(v_{\zeta} \mid \phi\right)=\left(\frac{\eta-1}{2}+3\left(h-\frac{\zeta}{2 \eta}-1\right)+2\left(h-2-\frac{\zeta}{2 \eta}\right)+\zeta-\left(2+2 \eta \frac{\zeta}{2 \eta}\right)\right) .
$$

Case 3. If $\zeta \equiv((\eta+5) / 2),((\eta+7) / 2), \ldots, \eta(\bmod 2 \eta)$,

$$
r_{2}\left(v_{\zeta} \mid \phi\right)=\left(\frac{3 \eta+3}{2}+3\left(h-1-\frac{\zeta}{2 \eta}\right)+2\left(h-2-\frac{\zeta}{2 \eta}\right)-\zeta+\left(2 \eta \frac{\zeta}{2 \eta}\right)\right)
$$

Case 4. If $\zeta \equiv 1(\bmod 2 \eta)$,

$$
r_{2}\left(v_{\zeta} \mid \phi\right)=\left(\frac{\eta-1}{2}+3\left(h-\frac{\zeta}{2 \eta}-1\right)+2\left(h-2-\frac{\zeta}{2 \eta}\right)+\zeta-\left(2 \eta \frac{\zeta}{2 \eta}\right)\right) .
$$

Case 5 . If $\zeta \equiv \eta+2, \eta+3, \ldots,((3 \eta+3) / 2)(\bmod 2 \eta)$,

$$
r_{2}\left(v_{\zeta} \mid \phi\right)=\left(\frac{\eta-3}{2}+3\left(h-\frac{\zeta}{2 \eta}-1\right)+2\left(h-2-\frac{\zeta}{2 \eta}\right)+\zeta-\left(2 \eta \frac{\zeta}{2 \eta}\right)-\eta+z\right)
$$

where $z=-2$ when $\zeta \equiv 7(\bmod 2 \eta)$ and, otherwise, $z=0$.
Case 6. If $\zeta \equiv 0,((3 \eta+5) / 2),((3 \eta+7) / 2), \ldots, 2 \eta-$ $1(\bmod 2 \eta)$,

$$
r_{2}\left(v_{\zeta} \mid \phi\right)=\left(\frac{5 \eta+5}{2}+3\left(h-\frac{\zeta}{2 \eta}-1\right)+2\left(h-2-\frac{\zeta}{2 \eta}\right)-\zeta+\left(2 \eta \frac{\zeta}{2 \eta}\right)\right) .
$$

Case 7. If $\zeta \equiv \eta+1(\bmod 2 \eta)$, 


$$
r_{2}\left(v_{\zeta} \mid \phi\right)=\left(\frac{3 \eta+5}{2}+3\left(h-\frac{\zeta}{2 \eta}-1\right)+2\left(h-2-\frac{\zeta}{2 \eta}\right)-\zeta+\left(2 \eta \frac{\zeta}{2 \eta}\right)\right) .
$$

Hence, it follows from the above discussion that $\operatorname{dim}\left(L_{S}(\eta, h)\right) \leq 2$ because all the vertices of $L_{S}(\eta, h)$ have unique representations with respect to resolving set $\phi$. For reverse inequality that $\operatorname{dim}\left(L_{S}(\eta, h)\right) \geq 2$, by contradiction, our assertion becomes $\operatorname{dim}\left(L_{S}(\eta, h)\right)<2$, implying $\operatorname{dim}\left(L_{S}\right.$ $(\eta, h))=1$, and it is not possible because only the path graph exists having the metric dimension 1 . All discussion concluding that when $\eta \geq 5$ (odd) and $h \geq 2$,

$$
\operatorname{dim}\left(L_{S}(\eta, h)\right)=2 \text {. }
$$

\section{Conclusion}

We found the metric dimension of some chemical networks ortho-, meta-, and para-polyphenyl chains constructed with base graph $C_{\eta}$ and sun graph $S_{\eta}$, and these networks have metric dimension $\operatorname{dim}(O(\eta, h))=\operatorname{dim}(M(\eta, h))=h$ and $\operatorname{dim}(L(\eta, h))=2$.

\section{Data Availability}

The data used to support the findings of this study are included within the article.

\section{Conflicts of Interest}

The authors declare that there are no conflicts of interest.

\section{Authors' Contributions}

All the authors contributed equally to prepare this article.

\section{Acknowledgments}

The authors extend their appreciation to the Deanship of Scientific Research at King Saud University for funding this work through Research Group no. RG-1441-453.

\section{References}

[1] P. J. Slater, "Leaves of trees, proceeding of the 6th southeastern conference on combinatorics, graph theory, and computing," Congressus Numerantium, vol. 14, pp. 549-559, 1975.

[2] F. Harary and R. A. Melter, "On the metric dimension of a graph,” Ars Combinatoria, vol. 2, pp. 191-195, 1976.

[3] P. J. Cameron and J. H. Van Lint, Designs Graphs, Codes and Their Links, Cambridge University Press, Cambridge, UK, 1991.

[4] G. Chartrand, L. Eroh, M. A. O. Johnson, and R. Ortrud, "Resolvability in graphs and the metric dimension of a graph," Discrete Applied Mathematics, vol. 105, no. 1-3, pp. 99-113, 2000.

[5] S. Khuller, B. Raghavachari, and A. Rosenfeld, "Landmarks in graphs," Discrete Applied Mathematics, vol. 70, no. 3, pp. 217-229, 1996.
[6] A. Sebö and E. Tannier, "on metric generators of graphs," Mathematics of Operations Research, vol. 29, no. 2, pp. 383393, 2004.

[7] P. Erdös and P. R. Alfréd, "On two problems of information theory," Magyar tudomanyos akademia matematikai kutato intezetenek kozlemenyei, vol. 8, pp. 229-243, 1963.

[8] B. Lindström, "On a combinatory detection problem I. I. Magyar Tud. Akad. Mat. Kutat \& oaccute,” Int Kazi, vol. 9, pp. 195-207, 1964.

[9] S. Imran, M. K. Siddiqui, and M. Hussain, "Computing the upper bounds for the metric dimension of cellulose network," Applied Mathematics E-Notes, vol. 19, pp. 585-605, 2019.

[10] M. K. Siddiqui and M. Imran, "Computing the metric and partition dimension of $\mathrm{H}-\mathrm{Naphtalenic}$ and $\mathrm{VC5C7}$ nanotubes," Journal of Optoelectronics and Advanced Materials, vol. 17, pp. 790-794, 2015.

[11] F. Simonraj and A. George, "On the metric dimension of silicate stars," ARPN Journal of Engineering and Applied Sciences, vol. 10, no. 5, pp. 2187-2192, 2015.

[12] Z. Hussain, M. Munir, M. Choudhary, and S. M. Kang, "Computing metric dimension and metric basis of $2 \mathrm{D}$ lattice of alpha-boron nanotubes," Symmetry, vol. 10, no. 8, 2018.

[13] P. Manuel, B. Rajan, I. Rajasingh, and C. Monica, "On minimum metric dimension of honeycomb networks," Journal of Discrete Algorithms, vol. 6, pp. 20-27, 2008.

[14] A. Ahmad, M. Baca, and S. Sultan, "Computing the metric dimension of kayak paddles graph and cycles with chord," Proyecciones (Antofagasta), vol. 39, no. 2, pp. 287-300, 2020.

[15] M. Ali, G. Ali, M. Imran, A. Q. Baig, and M. K. Shafiq, "On the metric dimension of möbius ladders," Ars Combinatoria, vol. 105, pp. 403-410, 2012.

[16] M. Ali, G. Ali, U. Ali, and M. T. Rahim, "On cycle related graphs with constant metric dimension," Open Journal of Discrete Mathematics, vol. 2, pp. 21-23, 2012.

[17] S. Hayat, A. Khan, M. Y. H. Malik, M. Imran, and M. K. Siddiqui, "Fault-tolerant metric dimension of interconnection networks," IEEE Access, vol. 8, pp. 145435-145445, 2020.

[18] M. Imran, A. Q. Baig, S. A. Bokhary, and I. Javaid, "On the metric dimension of circulant graphs," Applied Mathematics Letters, vol. 25, no. 3, pp. 320-325, 2012.

[19] D. Kuziak, J. A. Rodríguez-Velázquez, and I. G. Yero, "On the strong metric dimension of product graphs," Electronic Notes in Discrete Mathematics, vol. 46, pp. 169-176, 2014.

[20] J. B. Liu, M. F. Nadeem, H. M. A. Siddiqui, and W. Nazir, "computing metric dimension of certain families of toeplitz graphs,” IEEE Access, vol. 7, pp. 126734-126741, 2019.

[21] J. B. Liu, A. Zafari, and H. Zarei, "Metric dimension, minimal doubly resolving sets, and the strong metric dimension for jellyfish graph and cocktail party graph," Complexity, vol. 2020, Article ID 9407456, 7 pages, 2020.

[22] M. F. Nadeem, M. Azeem, and A. Khalil, "The locating number of hexagonal möbius ladder network," Journal of Applied Mathematics and Computing, 2020.

[23] H. Raza, J.-B. Liu, and S. Qu, "On mixed metric dimension of rotationally symmetric graphs," IEEE Access, vol. 8, pp. 11560-11569, 2019.

[24] H. Raza and Y. Ji, "Computing mixed metric dimension in generalized petersen graph $P(n, 2)$," Frontiers in Physics, vol. 8, p. 211, 2020. 
[25] H. Raza, Y. Ji, and S. Qu, "On mixed metric dimension of some path related graphs," IEEE Access, vol. 8, pp. 188146188153, 2020.

[26] M. Somasundari and F. S. Raj, "Fault-tolerant resolvability of oxide interconnections," International Journal of Innovative Technology and Exploring Engineering (IJITEE), vol. 8, pp. 2278-3075, 2019.

[27] T. Vetrík and A. Ahmad, "Computing the metric dimension of the categorial product of some graphs," International Journal of Computer Mathematics, vol. 94, no. 2, pp. 363-371, 2017.

[28] I. G. Yero, "Vertices, edges, distances and metric dimension in graphs," Electronic Notes in Discrete Mathematics, vol. 55, pp. 191-194, 2016. 